\title{
Designs of Self-tuning Control Systems for Ships
}

\author{
Duc-Hung NGUYEN*, Jin-Seok PARK* and Kohei OHTSU**
}

\begin{abstract}
This paper treats of the applications of the pole assignment self-tuning control theory to the identification and design of ship's auto-pilot systems. An on-line estimation algorithm known as the Recursive Least Squares Algorithm is used to identify parameters of the control system. Two typical types of the pole assignment self-tuning control algorithms that can be linked to the on-line estimation algorithm to form self-tuning control systems for ships are introduced. The authors discuss how to choose the control parameters by the rules of thumb in the actual sea trials. The pole assignment self-tuning controllers designed to steer ships are verified by experiments aboard the training ship Shioji Maru of Tokyo University of Mercantile Marine. The aboard experiments result in the confirmation that it is possible to keep and to change the ship's course as desired by the designed controllers.
\end{abstract}

Keywords : Recursive least squares algorithm, forgetting factor, self-tuning pole assignment control, system poles, PID controller and higher order pole assignment controller.

\section{Introduction}

The states of sea vary time to time. Therefore, a ship at seas has always been required to have an auto-pilot with a good performance for the purpose of safe and economical navigation. In recent years, many authors have indicated that it is important for a marine auto-pilot system to furnish with some adaptive tuning mechanisms. The self-tuning design method has given an effective control processing algorithm to a ship with time-varying maneuvering coefficients.

The basic idea of the self-tuning system is to construct an algorithm that automatically changes its parameters to meet a particular requirement or situation. The algorithm is referred to as an adjustment mechanism that monitors the system in operating and adjusts the coefficients of the corresponding controller to maintain a required performance ${ }^{(3)}$.

The self-tuning control theory was proposed in the form of minimum variance control based on an on-line recursive least square identification method by K.J. Astrom et al. at first. C.G. Kallstrom et al. applied the theory to an auto-pilot system for tankers. H. Mizuno et al. also developed an adaptive auto-pilot system using the self-tuning control theory. However, one disadvantage was that the minimum variance control law intrinsically did not take into account of rudder motion.

P.E. Wellstead et al. proposed one method that overtook it. According to this theory, a designer can arbitrarily assign system poles. Viewing this from designing of marine auto-pilot system, it means to be possible

(1) to give a desired maneuverability to a ship's course keeping motion and

* Student member : Graduate School of Tokyo University of Mercantile Marine

(2-1-6 Etchyujima, Koto-ku, Tokyo, 135-8533)

***Member : $\quad$ Tokyo University of Mercantile Marine

(2-1-6 Etchyujima, Koto-ku, Tokyo, 135-8533) 
(2) to change her course with desired response characteristics.

The authors planned

(1) to design a new auto-pilot system to be able to keep and to change the ship's course having appropriate maneuvering characteristics and to track along a given trajectory and

(2) to develop a new automatic berthing system and dynamic positioning one through a main engine and some side thrusters besides of rudder, using the framework of the pole assignment type of self-tuning control theory.

As the first step to realize those systems, the authors design a pole assignment type of auto-pilot system. And the main aims of this paper are put

(1) to actually test whether the pole assignment style of auto-pilot system as above stated is possible to be realized, using a small type of training ship;

(2) to prospect, if it can be realized, what problems exist in the theory to apply it to other general ships, before proceeding to a systematic study and assessment by simulations.

To formulate our problems as the framework of pole assignment type of self-tuning control theory, a brief survey on the self-tuning theory is made in Sections 2 and 3. In Section 4, the authors develop a prototype of auto-pilot system for a small training ship, carry out some actual sea trials and get some clues to generalize and to establish a consistent designing method of the pole assignment type of self tuning control to various types of ships.

\section{System Identification}

The identification method based on the self-tuning theory is a recursive type of least square identification one. In this section, the recursive algorithm to be solved at on-line is briefly discussed.

2.1 Discrete-time Linear Models of Ships

Ship's maneuvering characteristics using a rudder are considered a linear stochastic model with input and output signals. For generalization, the system with control inputs such as the rudder signal $u(t)$, and outputs such as the ship's course $y(t)$, subject to random sea disturbances $e(t)$ can be expressed by a linear difference equation

$$
A y(t)=B u(t-1)+C e(t)
$$

where $A, B$ and $C$ are polynomials of the $z^{-1}$ and defined as

$$
\begin{aligned}
& A\left(z^{-1}\right)=1+a_{1} z^{-1}+a_{2} z^{-2}+\cdots+a_{n} z^{-n} \\
& B\left(z^{-1}\right)=b_{0}+b_{1} z^{-1}+b_{2} z^{-2}+\cdots+b_{m} z^{-m} \\
& C\left(z^{-1}\right)=1+c_{1} z^{-1}+c_{2} z^{-2}+\cdots+c_{q} z^{-q}
\end{aligned}
$$

and $z^{-1}$ is the unit backward shift operator defined by $z^{-i}-y(t)=y(t-i)$. The model in (2.1) is known as an ARMAX (Auto-Regressive Moving Average eXogenous) model.

\subsection{Recursive Least Squares (RLS) Algorithm}

The first task of designing a self-tuning control system is to construct an appropriate on-line estimation algorithm to predict unknown parameters of the assumed model. In this paper, the following on-line procedure is used.

The ARMAX model in (2.1) is recalled. Using a matrix form, this model can be expressed

$$
y(t)=\phi^{T}(t) \theta+e(t)
$$

wher $\phi^{T}$ is a regressive vector consisting of measured input and output variables, defined by

$$
\Phi^{T}=[y(t-1), \cdots, y(t-n), u(t-1), \cdots, u(t-m-1), e(t-1), \cdots, e(t-q)]
$$

and $\theta$ is the vector of unknown parameters, defined by

$$
\theta^{T}=\left[-a_{1}, \cdots,-a_{n}, b_{0}, b_{1}, \cdots, b_{m}, c_{1}, \cdots, c_{q}\right]
$$

The RLS algorithm can be summarized as follows, 
At time step $t+1$ :

(i) Form $\phi(t+1)$ using the new data.

(ii) Form the prediction error $\varepsilon(t+1)$ using

$$
\varepsilon(t+1)=y(t+1)-\phi^{T}(t+1) \hat{\theta}(t)
$$

(iii) Form $\mathbf{P}(t+1)$ using

$$
\mathbf{P}(t+1)=\mathbf{P}(t)=\left(\mathbf{I}_{M}-\frac{\phi(t+1) \phi^{T}(t+1) \mathbf{P}(t)}{1+\phi^{T}(t+1) \mathbf{P}(t) \phi(t)}\right)
$$

If the forgetting factor technique is used, the equation (2.9) becomes

$$
\mathbf{P}(t+1)=\lambda^{-1} \mathbf{P}(t)\left[\mathbf{I}_{M}-\frac{\phi(t+1) \phi^{T}(t+1) \mathbf{P}(t)}{\lambda+\phi^{T}(t+1) \mathbf{P}(t) \phi(t)}\right)
$$

(iv) Update $\hat{\theta}(t)$ using

$$
\hat{\theta}(t+1)=\hat{\theta}(t)+\mathbf{P}(t+1) \phi(t+1) \varepsilon(t+1)
$$

(v) Wait for the next time step to elapse and loop back to step (i).

It should be noticed that $\mathbf{P}$ is the covariance matrix initially chosen as $\alpha \mathbf{I}_{M}(\alpha=$ a large number, for example, 1,000$)^{(4)}$, the subscripted $M$ shows the number of unknown parameters, and $\lambda$ is the forgetting factor chosen in the range of $(0.98 \text { to } 0.998)^{(7)}$.

\section{Self-tuning Control Systems}

After the on-line identification, some control signal is calculated for the rudder command sig nal. In this paper, two typical types of pole assignment self-tuning controllers, proposed by P.E. Wellstead et al., which can be linked to the above-mention on-line estimation algorithm to form a self-tuning or adaptive control system for ships are considered for the reason discussed in Section 1 .

\subsection{Pole Assignment Control (PAC) Algorithm}

When an ARMAX model of ship as the eq. (2.1) is considered, the PAC algorithm is calculated as follows. Assuming that the controller is of the form

$$
F u(t)=H r(t)-G y(t)
$$

where $r(t)$ is the reference signal or setting course, and combining the controller and the system equation (2.1) yields the closed-loop description

$$
\left(F A+z^{-1} B G\right) y(t)=z^{-1} B H r(t)+C F e(t)
$$

The basic technique of the PAC algorithm is that poles of the closed-loop equation are assigned to the desired locations specified by a desired pole set polynomial $\mathbf{T}$, given by

$$
\mathbf{T}=1+t_{1} z^{-1}+\cdots+t_{n t} z^{-n t}
$$

The control gains can be determined by assigning the closed-loop poles to the desired locations

$$
F A+z^{-1} B G=\mathbf{T} C
$$

where $F, G$ and $H$ are defined by

$$
\begin{aligned}
& F=1+f_{1} z^{-1}+f_{2} z^{-2}+\cdots+f_{f} z^{-f} \\
& G=g_{0}+g_{1} z^{-1}+g_{2} z^{-2}+\cdots+g_{g} z^{-g} \\
& H=h_{0}+h_{1} z^{-1}+h_{2} z^{-2}+\cdots+h_{h} z^{-h}
\end{aligned}
$$

with the condition is that degrees of $f, g$ should be selected as $f=m, g=n-1(n \neq 0)$ provided that $A, B$ have no common zeros and $n_{t} \leq n+m-q$. Inserting (3.4) into (3.2), gives

$$
y(t)=[H B / \mathbf{T} C] r(t-1)+[F / \mathbf{T}] e(t)
$$

where the noise polynomial $C$ has been cancelled in the disturbance term by selecting the $H$-polynomial as

$$
H=C[\mathbf{T} / B]_{z=1}
$$


Then (3.5) can be written as

$$
y(t)=[\mathbf{T} / B]_{z}=1[B / \mathbf{T}] r(t-1)+[F / \mathbf{T}] e(t)
$$

3.2 PID Type of PAC Algorithm

Let's apply this PAC algorithm for designing a simple self-tuning controller known as a PID (Proportional, Integral and Derivative) controller in the form

$$
u(t)=\frac{\left(g_{0}+g_{1}+g_{2}\right) r(t)-\left(g_{0}+g_{1} z^{-1}+g_{2} z^{-2}\right) y(t)}{1-z^{-1}}
$$

where $r(t)$ is the setting course, $y(t)$ is the yaw angle and $u(t)$ is the rudder angle. Then, it must be assumed that the ship model has a special structure

$$
y(t)=\frac{b_{0} z^{-1}}{1+a_{1} z^{-1}+a_{2} z^{-2}} u(t)
$$

It becomes easy to design the PAC, when the desired pole set polynomial $\mathbf{T}$ can be specified by

$$
\mathbf{T}=1+t_{1} z^{-1}+t_{2} z^{-2}
$$

where its coefficients might be calculated by the following vibration equations

$$
\begin{aligned}
& t_{1}=-2 \exp \left(-\xi \omega_{n} \tau_{s}\right) \cos \left\{\tau_{s} \omega_{n}\left(1-\xi^{-2}\right)^{1_{s}}\right\} \\
& t_{2}=\exp \left(-2 \xi \omega_{n} \tau_{s}\right)
\end{aligned}
$$

where $\xi, \omega_{n}, \tau_{s}$ are the damping factor, natural frequency and sampling time, respectively.

Finally, the coefficients of the $G$-polynomial can be determined by

$$
\begin{aligned}
& g_{0}=\left[t_{1}+\left(1-a_{1}\right)\right] /\left[b_{0}\right] \\
& g_{1}=\left[t_{2}+\left(a_{1}-a_{2}\right)\right] /\left[b_{0}\right] \\
& g_{2}=\left[a_{2}\right] /\left[b_{0}\right]
\end{aligned}
$$

\subsection{Higher Order Type of PAC Algorithm}

As the other typical pole assignment controller which is designed here is a higher order type controller having an ARX model with $n=3, m=2, q=0$, $\mathbf{T}$ as the equation (3.10), $f=2$ and $g=$ 2. Using the RLS algorithm, unknown parameters of $A, B$ can be estimated. The coefficients of $F$ and $G$ are resulted in the following system of linear equations

$$
\mathbf{A} \Phi_{c}=\mathbf{b}
$$

where $\mathbf{A}$ is a matrix formed by the estimated parameters of $A$ and $B$, and

$$
\begin{aligned}
& \Phi c^{T}=\left[f_{1}, f_{2}, g_{0}, g_{1}, g_{2}\right] \\
& \mathbf{b}^{T}=\left[\left(t_{1}-a_{1}\right),\left(t_{2}-a_{2}\right),\left(-a_{3}\right), 0,0\right]
\end{aligned}
$$

Solving this system of equations, choosing $H$-polynomial such that $H=\left[\left(1+t_{1}+t_{2}\right) /\left(b_{0}+b_{1}+b_{2}\right)\right]$ with $C=1$, and using the deviation of the setting course and the yaw angle ${ }^{(11)}$, the rudder angle signal might be calculated by

$$
u(t)=-G y(t) / F
$$

However, using the formulae (3.8) and (3.15) to compute the rudder angle signal, an issue of dividing by zero arises from that the parameter $b_{0}$ in the denominator as in (3.13) becomes zero or the matrix $\mathbf{A}$ in (3.14) is singular. Therefore, the controller is not stable. The solution is that the parameter $b_{0}$ could arbitrarily be selected as a constant while other parameters of the system should be estimated by the RLS algorithm. In the next section, the choice of $b_{0}$ is discussed in more details and illustrated by experiments.

Another issue arises from the assignment of the system poles to the desired poles as shown in (3.4). The desired poles in (3.3) effects on the stability of the controller. If the coefficients of the $\mathbf{T}$-polynomial are selected appropriately such that the desired poles are located inside the circle with the radius one in the complex $z$-plane the system would be considered stable ${ }^{(10)}$. When implementing the experiments aboard the actual ship, the natural frequency, damping factor and sampling time should be chosen appropriately such that the poles of the system satisfy the condition of being inside the unit circle. The choices of the system poles are discussed in more details 
and illustrated by experiments in the next section.

\section{Experiments and Results}

\subsection{The Training Ship, Shioji Maru}

Experiments of designed self-tuning controllers were implemented aboard the training ship Shioji Maru of Tokyo University of Mercantile Marine. The ship was built for the purposes of training seafarer's works, implementing experiments and research related to ship and survey of marine traffic. Table 1 shows the principal particulars of the Shioji Maru.

Table 1 Principal particulars of the Shioji Maru

\begin{tabular}{|l|c|l|c|}
\hline Length between perpendiculars & $46.00 \mathrm{~m}$ & Breadth & $10.00 \mathrm{~m}$ \\
\hline Depth & $2.08 \mathrm{~m}$ & Displacement & $717.52 \mathrm{t}$ \\
\hline Gross Tonnage & $425.00 \mathrm{t}$ & Propeller & $\mathrm{CPP}$ \\
\hline Bow thrust & $2.4 \mathrm{t}$ & Stern thrust & \\
\hline
\end{tabular}

\subsection{Experiments and Results}

Before carrying out the aboard experiments, the RLS algorithm was verified by the simulation programs in MATLAB and FORTRAN codes using the data available. The effects of the initial covariance matrix and forgetting factor were also tested.

Experiments of designed controllers were implemented aboard the Shioji Maru. The control programs executed by YEWMAC line computers can be illustrated by the flowchart in Fig. 1. Many experiments were implemented aboard the Shioji Maru for investigating how the RLS algorithm give the estimated values of the system parameters and how designed controllers run. In this paper, two types of experiments are tested. From the following, the PID type of controller is referred to as PIDC, high-order one, as the HOPAC. Three representative experiments are described below.

\subsubsection{Experiment №. 1 : PIDC for Course -} keeping-Choice of the Parameter $b_{0}$

The designed auto-pilot was used to steer the ship as a course-keeping controller. The system model was of the form in (3.9). The control gains were calculated by (3.12). The rudder angle was calculated by (3.8) with assumption that $r(t)$ would be zero and $y(\mathrm{t})$ would be the deviation of the setting course and the true course. But we must note that the parameter $b_{0}$ in (3.13) might be zero during the on-line estimation as the result, the equation (3.13) might

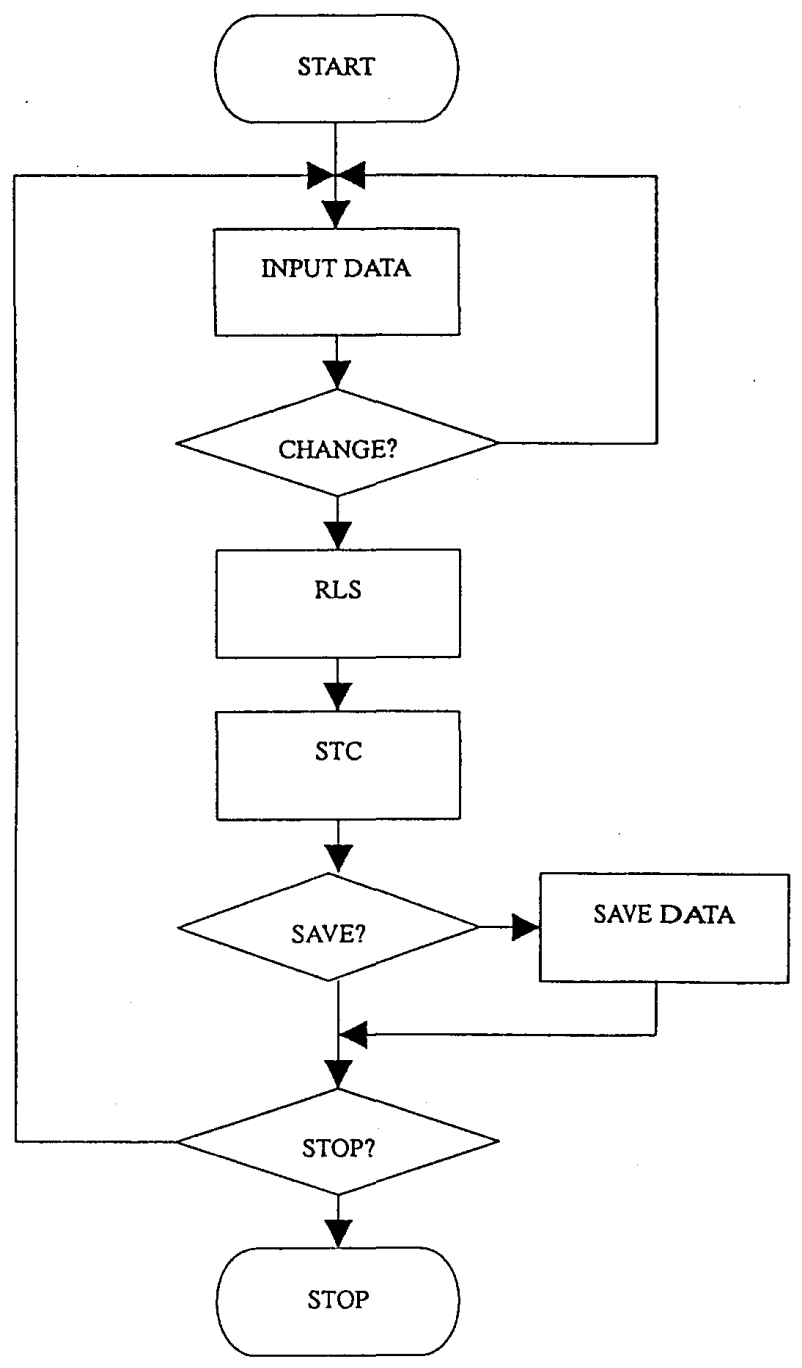

Figure 1 Flowchart of control programs 


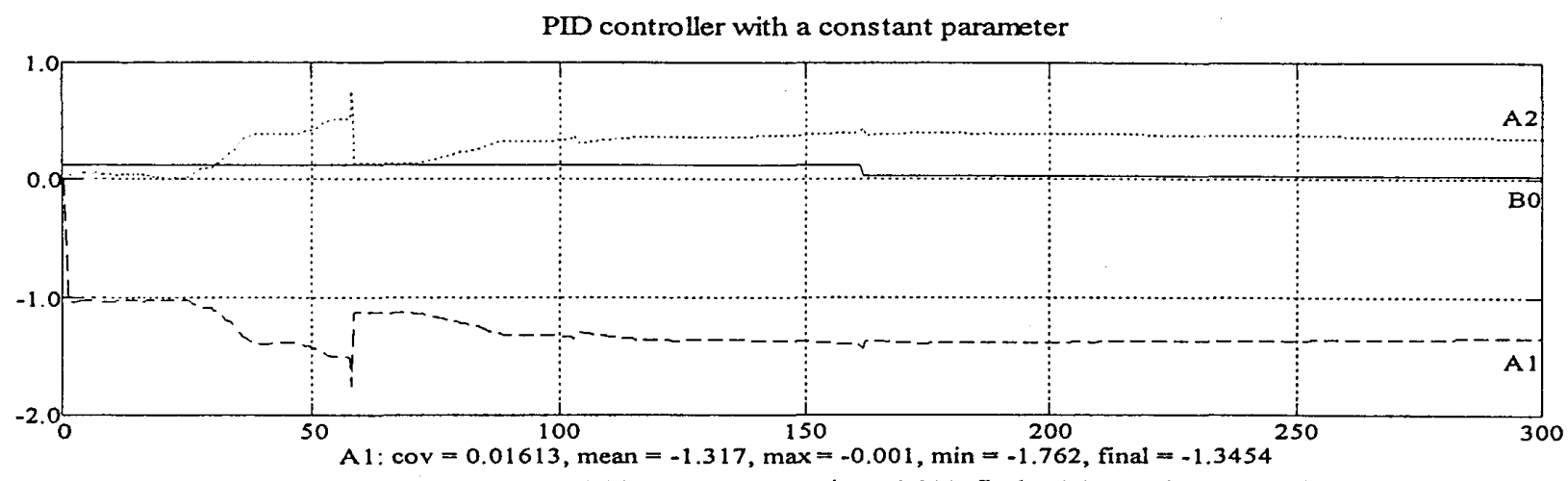

A2: $\operatorname{cov}=0.01329$, mean $=0.32, \max =0.762, \min =-0.001$, final $=0.394$ and $B 0=0.12 / 0.03$

Time series of estimated parameters with samp ling time $=0.5$

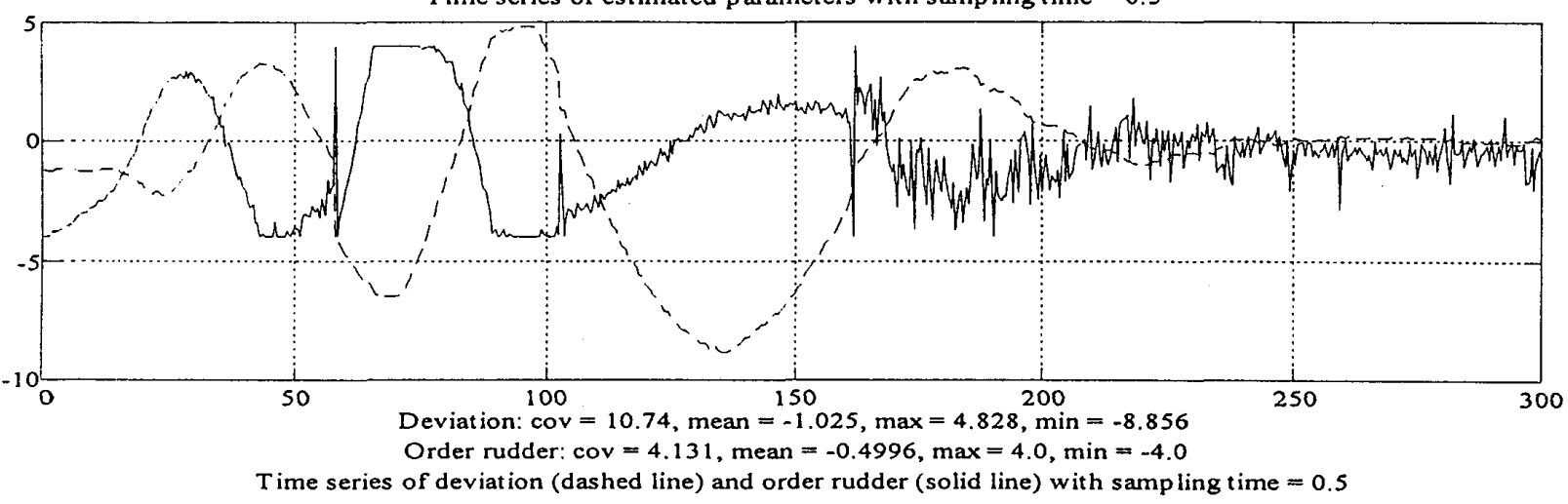

$\mathrm{NF}=0.12$

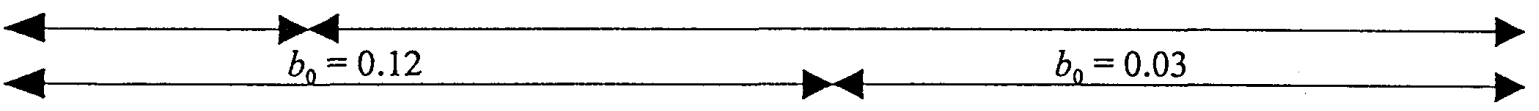

Setting course $=85$ degree

Forgetting factor $(\mathrm{FF})=0.995$

Damping factor $(\mathrm{DF})=0.6$

Natural frequency (NF) $=0.12,0.06$ at $t=60$

Initial covariance matrix $=1,000 \mathbf{I}_{2}$

Average speed $=10$ knots

Figure 2 Time series of the experiment №. 1 (choice of $b_{0}$ )

be singular. In order to avoid this difficulty, we assumed that the parameter $b_{0}$ would beforehand be fixed to an appropriate definite value and whose value was determined in the actual sea testing by rules of thumb. And then, the damping factor, the natural frequency were also selected in the actual tests.

Fig. 2 shows the experiment that was carried out for determining the value of $b_{0}$. It demonstrates the time series of estimated parameters, rudder angle and deviation. In this figure, from $t=0$ to $160\left(b_{0}=0.12\right)$, coefficients were not proper since the rudder angle was rather great. But from $t=160\left(b_{0}=0.03\right)$, it can be seen that the parameters converged at stable values, and the rudder angle became smaller and the ship course was kept stable as desired. In this case, the natural frequency was changed, but this choice is discussed below.

Thus in the experiments of PID controller which were carried out in the later, the parameter $b_{0}$ would be fixed to 0.03 .

4.2.2 Experiment No.2 : PIDC for Course-keeping - Choices of the Forgetting Factor and System Poles

As the next step we proceeded the choices of the forgetting factor, system coefficients $\omega_{n}$ and $\xi$. At that time, the parameter $b_{0}$ was set to 0.03 . Fig. 3 shows the time series of the experiments. From $t=0$ to 100 , the ship was steered by her auto-pilot and from $t=100$, the ship was steered by the PIDC. After that, the tests for the forgetting factor $\lambda$ and some values of sys- 
PID controller with a constant (choices of coefficients)

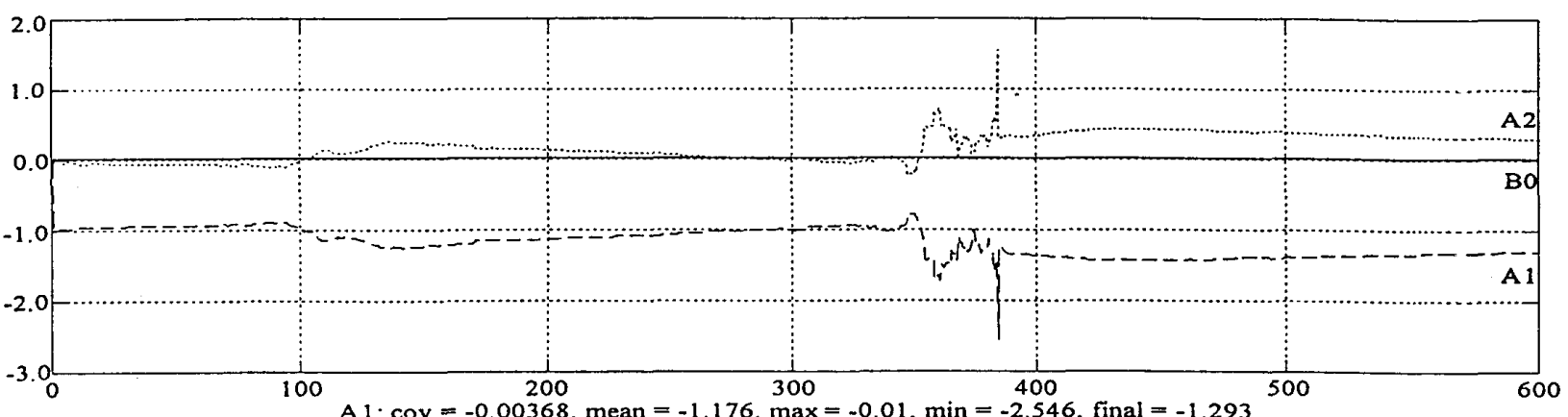

$\mathrm{A} 2: \operatorname{cov}=0.003599$, mean $=1.495$, mean $=0.1767, \max =1.544, \min =-0.235$, final $=0.2924$ and $\mathrm{B} 0=0.03$

Time series of estimated parameters with sampling time $=0.5$
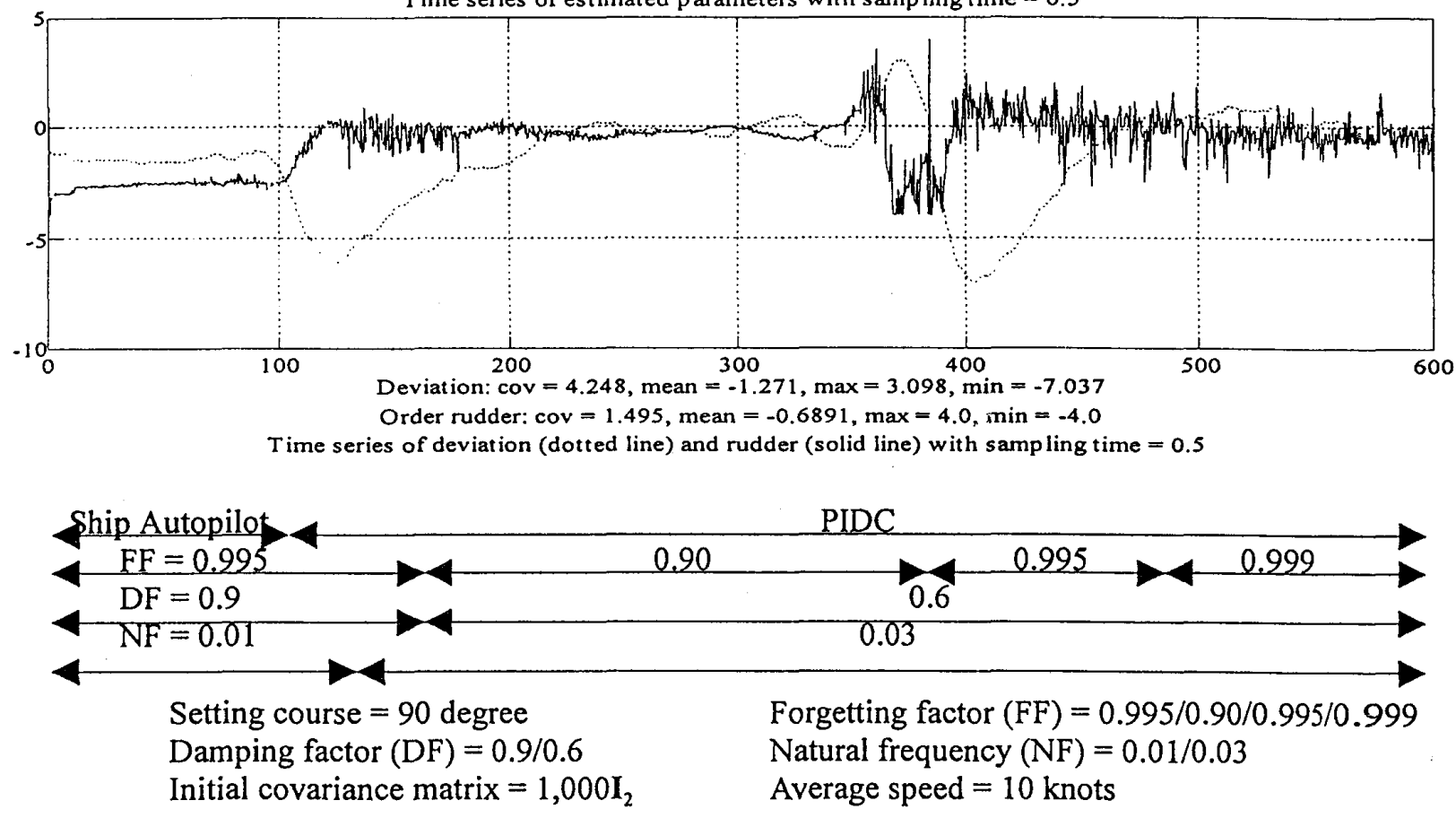

Figure 3 Time series of the experiment No. 2 (choices of coefficients)

tem poles were carried out.

Choices of forgetting factor: As above-mentioned, the forgetting factor $\lambda$ effects on the convergence of the estimated parameters. According to P.E. Wellstead et al. ${ }^{(4)}$, the forgetting factor would be in the range of 0.90 and 1.0. During implementation of experiments, the forgetting factor was chosen in the range of 0.90 to 0.998 . When the forgetting factor was changed, the estimated parameters were effected, and the control gains were also changed. Experiments were implemented for verifying the effect of the forgetting factor on the estimated parameters and control gains. The tests in Fig. 3 show the effect of the forgetting factor. The forgetting factor was initially set to 0.995 , and was changed to 0.90 at $t=180$, was returned to 0.995 at $t=385$ and was changed to 0.999 at $t=480$. When the forgetting factor was reduced its value, the change of the rudder angle became large, so it effected on the sensibility and stability of the controllers. If the chosen forgetting factor was less than 0.98 , the control became unstable and the rudder angle was so great that it was difficult to maintain the ship course. When the forgetting factor was equal to or greater than 0.995 , the controller became stable. Therefore, the forgetting factor has been chosen as 0.995 for many experiments aboard the ship at the present.

Choices of coefficients of the desired pole set polynomial : As above-mentioned, the stability of 
a self-tuning control system was effected by the system poles (see Ref. 4 and 10 about the system poles and zeros in more details). When the assigned poles are appropriate, the controller become stable, in other words, the reference signal would be maintained as desired and in the steady-state condition. According to the stability analysis, the self-tuning control system will be stable only if the desired poles of the system are inside the unit circle on the complex $z$-plane for an example as shown in Fig. 5 .

The ordinary method to assign the appropriate positions of system poles is to implement some simulations. However, in this paper, these coefficients were appropriately chosen during implementing actual experiments. Fig. 3 shows the result of choices of the natural frequency $\omega_{n}$ and the damping factor $\xi$, from $t=0$ to $150, \omega_{n}=0.01$ and $\xi=0.9\left(p_{1}=0.9955+0.0022 \mathrm{i}, p_{2}=\right.$ $0.9955-0.0022 \mathrm{i}$, respectively $)$, from $t=150, \omega_{n}=0.03$ and $\xi=0.9\left(p_{1}=0.9866+0.0065 \mathrm{i}, p_{2}=0.9866-\right.$ $0.0065 \mathrm{i}$, respectively), and from $t=180, \omega_{n}=0.03$ and $\xi=0.6$ ( $p_{1}=0.9910+0.0119 \mathrm{i}, p_{2}=0.9910-0.0119$ i, respectively). Fig. 5 demonstrates the locations of each pole in the corresponding case of $\omega_{n}=$ 0.01 and $\xi=0.9$.

As the results of these experiments, it is confirmed that the designer can select the desired ship response as far as the system pole locates inside unit circle.

\subsubsection{HOPAC for Course-keeping}

We have not yet carried out such systematic experiments with respect to this type of auto-pilot system as the above-mentioned PIDCs. Therefore, one example is demonstrated here.

Higher-order PA controller with a constant parameter

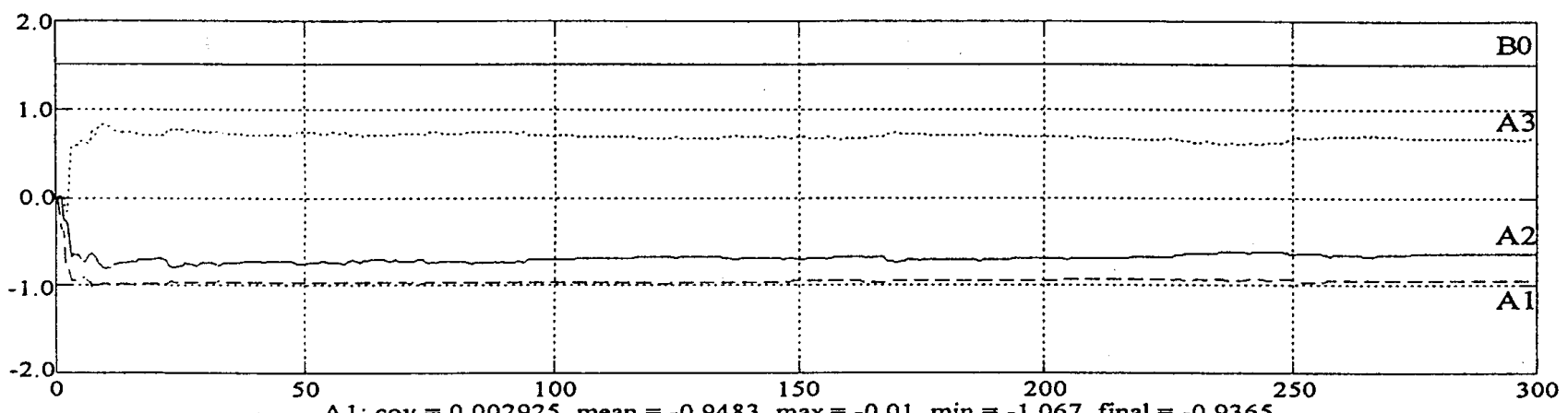

A 1 : $\operatorname{cov}=0.002925$, mean $=-0.9483, \max =-0.01, \min =-1.067$

A. $: \operatorname{cov}=0.003916$, mean $=-0.69872, \max =-0.01, \min =-0.1697$, final $=-0.628$

A 3: $\operatorname{cov}=0.006$, mean $=0.6858, \max =0.831, \min =-0.1697$, final $=0.6588$ and $\mathrm{B} 0=1.5$

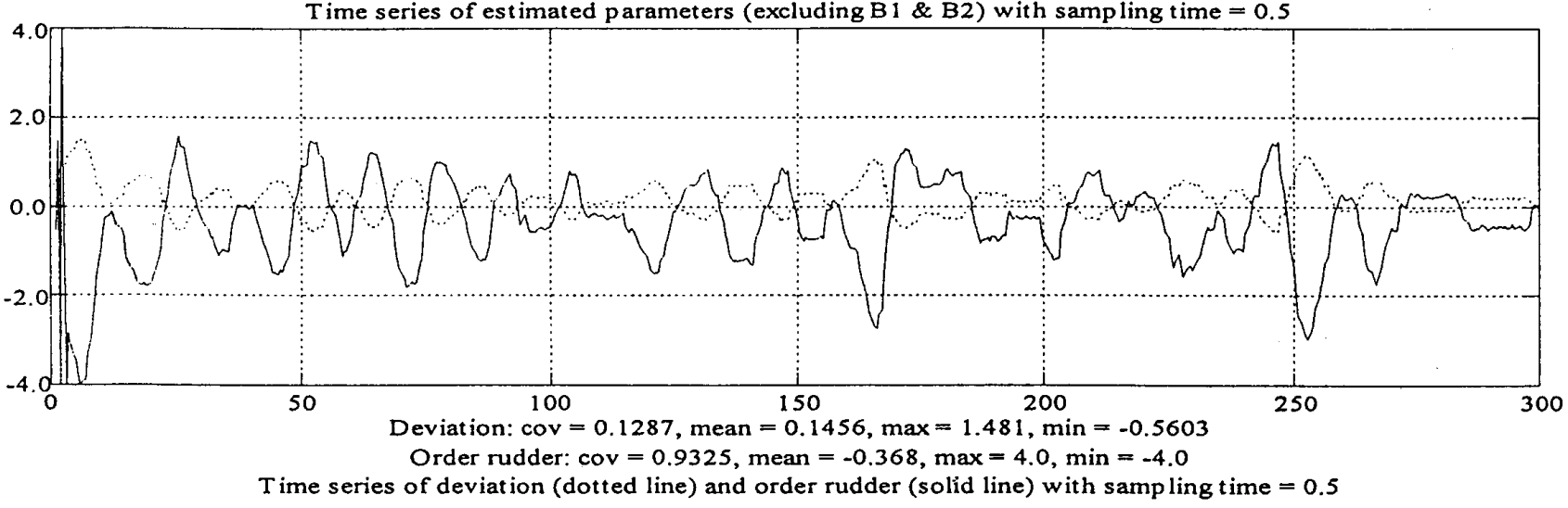

HOPAC

Ship Autopilot

Setting course $=10$ degree

Damping factor $(\mathrm{DF})=0.6$

Initial covariance matrix $=1,000 \mathbf{I}_{5}$
Forgetting factor $(\mathrm{FF})=0.995$

Natural frequency (NF) $=0.03$

Average speed $=10.19$ knots

Figure 4 Time series of the experiment №. 3 
Table 2 Summary of experiments

\begin{tabular}{|l|c|c|c|c|c|}
\hline \multicolumn{1}{|c|}{ Controllers } & $\begin{array}{c}\text { Estimated } \\
\text { Parameters }\end{array}$ & $\begin{array}{c}\text { Setting } \\
\text { Course }\end{array}$ & $\begin{array}{c}\text { Natural } \\
\text { Frequency }\end{array}$ & $\begin{array}{c}\text { Damping } \\
\text { Factor }\end{array}$ & $\begin{array}{c}\text { Forgetting } \\
\text { factor \& } \alpha\end{array}$ \\
\hline $\begin{array}{l}\text { PIDC (for course- keep- } \\
\text { ing), choice of the } \\
\text { parameter } b_{0}\end{array}$ & $\begin{array}{c}a_{1}, a_{2} \\
b_{0}=0.12 \& \\
0.03\end{array}$ & 85 & 0.12 & 0.6 & 0.995 \\
& & & & & \\
\hline $\begin{array}{l}\text { PIDC (for course- keep- } \\
\text { ing), choices of the sys- } \\
\text { tem poles }\end{array}$ & $\begin{array}{c}a_{1}, a_{2} \\
b_{0}=0.03\end{array}$ & 90 & $0.01 / 0.03$ & $0.9 / 0.6$ & $\begin{array}{c}0.995 / 0.90 / \\
1,000\end{array}$ \\
\hline $\begin{array}{l}\text { HOPAC (for course- } \\
\text { keeping) }\end{array}$ & $\begin{array}{c}a, a_{2}, a_{3} \\
b_{0}=1.5\end{array}$ & 10 & 0.03 & 0.6 & 0.995 \\
& $b_{1}$ & & & & 1,000 \\
\hline
\end{tabular}

The parameter $b_{0}$ was chosen as a constant for preventing the calculation of rudder signal from dividing by zero. The control gains of the controller were obtained by solving the equation (3.14). The rudder signal was calculated by (3.15). Fig. 4 gives one example of the time series of parameters of the controller. The estimated parameters converged at stable values. In this case, the parameter $b_{0}$ was chosen as 1.5 , the sampling time $=0.5$, the natural frequency $=0.03$ and the damping factor $=0.6$. The system poles were located at values $\left(p_{1}=0.9910+0.0119 \mathrm{i}, p_{2}=0.9910-0.0119 \mathrm{i}\right)$.

It is certainly confirmed that the HOPAC might show good performances if all parameters are well tuned. However, it is necessary to carry out further study about this type of auto-pilot.

\subsection{Summary of All Experiments}

The summary of experiments is in Table 2 . These experiments show the results that are considered the best choice of the parameters at the present.

\section{Conclusions}

From the brief survey of the self-tuning control theory and its application to the system identification and designing self-tuning controllers for ships and successful experiments, main conclusions could be summarized below.

1) It was found that the on-line RLS algorithm could be applied to estimation of unknown parameters of a discrete-time linear model of ship. The estimated values were considerably effected by initial settings of covariance matrix, forgetting factor and system poles, especially, $b_{0}$ was a quite important parameter. By appropriate selection of their parameters, the algorithm gave a good convergence of the mod-

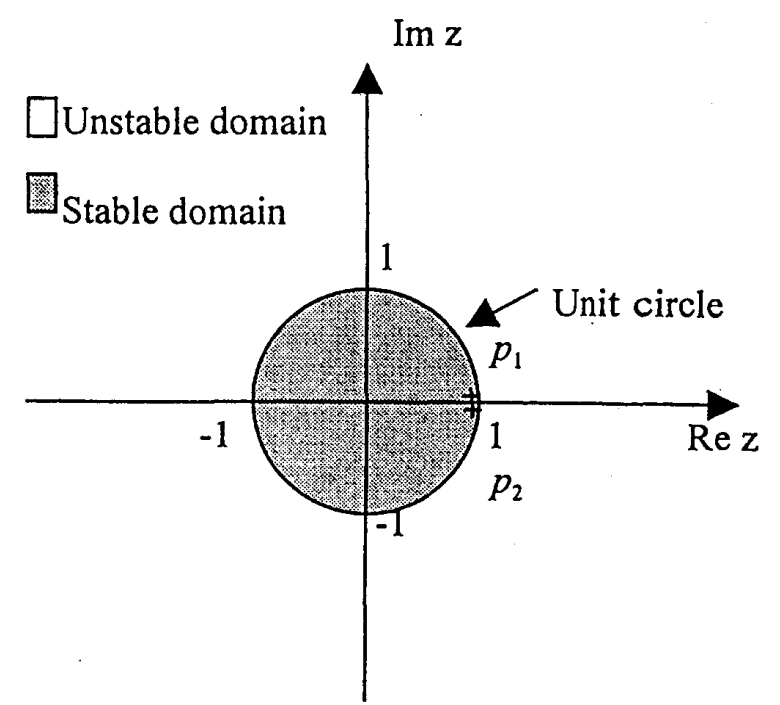

Figure 5 Example of the system poles inside the unit circle(for the exp. No. 2) 
el parameters and a good performance of the control system.

2) The feasibility of using self-tuning techniques for ship steering was explored. It was found that the computational requirements were reasonable and the self-tuning controllers could be applied to ship steering. The ship heading was rather stable when the estimated parameters converged at stable values.

3) In comparison with the actual ship's auto-pilot (for examples, see Fig. 3, from $t=0$ to 100 and Fig. 4 , from $t=225$ to 300 , the ship was steered by her auto-pilot), the designed self-tuning controllers worked well. The rudder angle signal generated by the self-tuning controllers was sufficiently small, so it was easy to keep the ship course stable as desired.

\section{Acknowledgement}

The sincere acknowledgement would be expressed to Captain H. Imo, Captain H. Fukui, Chief Engineer T. Hotta and other crew members of the training ship Shioji Maru for their enthusiastic helpings in the experiments.

\section{References}

(1) H. Mizuno et al., Route Tracking System by Adaptive Auto-pilot. Proc. of CAMS'89, Copenhagen, 1989.

(2) K.J. Astrom, Theory and Applications of Adaptive Control - A Survey. Aumatica, 19(5), 471-86, 1983.

(3) Claes G. Kallstrom, Identification and Adaptive Control Applied to Ship Steering. Doctoral Thesis. Lund University, 1979.

(4) P.E. Wellstead and M.B. Zarrop, Self-tuning Systems - Control and Signal Processing. John Wiley \& Sons, 1991.

(5) Benjamin C. Kuo, Automatic Control Systems. Seventh editions. Prentice Hall, 1995.

(6) Thor I. Fossen, Guidance and Control of Ocean Vehicles. John Wiley \& Sons, 1994.

(7) Chang C. Hang, Tong H. Lee \& Weng K. Ho, Adaptive Control. Instrument Society of America, 1993.

(8) Kohei Ohtsu, Hiroyuki Oda \& Takeshi Iida, Challenge to Advanced Optimal Ocean Navigation System, in Japanese. Ship Motion and Its Control at Seas. The $13^{\text {th }}$ Marine Dynamics Symposium, Part 2. The Society of Naval Architecture of Japan, June 1997.

(9) Ioan Dore Landau, System Identification and Control Design. Prentice Hall, 1990.

(10) James A. Cadzow, Discrete-time Systems. Prentice Hall, 1973.

(11) C.J. Harris \& S.A. Billings, Self-tuning and Adaptive Control : Theory and Application. Peter Peregrinus Ltd., revised second edition 1985.

(12) E.M. Goodwin \& J.F. Kemp, Maritime Statistics Theory and Practice. Stanford Maritime London, 1979.

(13) Henry H. Hooyer, Behavior and Handling of Ships. Cornell Maritime Press, 1983.

(14) Report of Tank Test [Shioji Maru, Series No. 2957], IHI Ship Model Basin, Ishikawajima-Harima Heavy Industries Co., Ltd..

(15) Takeshi Ishibashi, Study Related to Automatic Maneuver Supporting Devices, in Japanese. Master Thesis, March 1997.

森本 隆(横河電子機器(侏)：パラメータ $b_{0}$ が 0 の場合は、セルフ・チューニング制御は不安定となるが、 
もし0の場合の解決は、いかに考えているのか。

Takashi MORIMOTO (Yokogawa Denshikiki Co., Ltd.) : In the case that the parameter $b_{0}$ would be zero, I suppose, the self-tuning controller might be unstable, if possible, how should the solution in this case be considered? (Translated by the authors)

Duc-Hung NGUYEN : Thank you for your discussion. As I mentioned above, the parameter $b_{0}$ in the case of the self-tuning controller was pre-specified as a constant for preventing the calculation of the control signals from dividing by zero as shown in the equations (3.13) and (3.14) and avoiding the control system from being unstable. In the case of the PID self-tuning controller, $b_{0}$ was fixed and chosen as 0.03 and in the case of the HOPAC, $b_{0}$ was fixed and chosen as 1.5. Other parameters are assumed unknown and should be estimated by the RLS algorithm. 